\title{
Review
}

Clinical

nephron

Practice

\section{The Evolution of Pediatric Continuous Renal Replacement Therapy}

\author{
Francesco Garzotto Monica Zanella Claudio Ronco \\ Department of Nephrology, Dialysis and Transplantation, International Renal Research Institute (IRRIV), \\ San Bortolo Hospital, Vicenza, Italy
}

\section{Key Words}

Continuous renal replacement therapy $\cdot$ Infants .

Extracorporeal therapy

\begin{abstract}
The provision of continuous renal replacement therapies (CRRT) to small children has generally required the adaptation of adult machines and modified operational characteristics. CRRT prescription for younger and smaller children versus adults differs significantly due to problems concerning the extracorporeal blood volume, the need for circuit blood priming, and the adaptation of machines designed for adult-sized patients. Moreover, the provision of renal replacement therapy to infants and neonates presents a unique problem: no more than $10-15 \%$ of their blood volume should be removed by the extracorporeal circuit to prevent hypotension and anemia. In 2012, a dedicated machine, i.e. the Cardio-Renal, Pediatric Dialysis Emergency Machine (CARPEDIEM), was developed and launched the 'fitted era' for pediatric CRRT. In this review, we analyze how CRRT techniques have evolved for pediatric application and describe the first in vivo application of the CARPEDIEM for the safe and efficacious provision of CRRT to infants.
\end{abstract}

(c) 2014 S. Karger AG, Basel

Changing Paradigms in Acute Kidney Injury: From Mechanisms to Management - Proceedings of the 5th Annual UAB-UCSD O'Brien Center Symposium (San Diego, Calif., USA, March 4, 2014).

\section{Introduction}

In an era in which debates on continuous renal replacement therapies (CRRT) are mainly focused on the dose, the timing of the initiation, the appropriate therapy, and duration, pediatric patients are a relatively neglected population. Pediatric CRRT management differs from that in adults with respect to patient size, the spectrum of diseases requiring therapy, and the lower margin of safety that needs to be balanced with treatment efficacy. The provision of CRRT to small children has generally been based on the adaptation of adult equipment and modified procedures that may predispose patients to additional risks. In this review, we trace the development and application of pediatric CRRT and describe the most recent machines that have been developed to manage critically ill pediatric patients.

\section{Epidemiology of Acute Kidney Injury and CRRT Use in Critically III Pediatric Patients}

The epidemiology of acute kidney injury (AKI) in pediatric patients has changed significantly over the last few decades. In the 1980s, primary renal disease, hemolytic uremic syndrome, sepsis, and burns were the most prevalent causes of AKI in neonates and children. In the last decade, AKI has most often resulted from complications of other systemic chronic diseases [1] (cardiac, oncologic, hepatic, or pulmonary), surgery for congenital heart disease, neonatal care, acute tubular necrosis, and bone marrow

\section{KARGER}

E-Mail karger@karger.com

www.karger.com/nec
C 2014 S. Karger AG, Basel

$1660-2110 / 14 / 1274-0172 \$ 39.50 / 0$
Francesco Garzotto

Department of Nephrology, Dialysis and Transplantation

International Renal Research Institute (IRRIV), San Bortolo Hospital

Viale Rodolfi, 37, IT-36100 Vicenza (Italy)

E-Mail f.garzotto@gmail.com 
and solid transplantation, as well as multiorgan dysfunction syndrome, nephrotoxic medications, and septic shock. Recent studies have indicated that up of $10 \%$ of all children in the Pediatric Intensive Care Unit (PICU) develop AKI. The presence of AKI, on admission or during the PICU stay, is independently associated with mortality and there is a direct correlation between mortality and progression of the RIFLE score [2]. Primarily the indications for renal replacement therapy in critically ill children are similar to those in adults and include fluid overload, AKI, electrolyte imbalances, sustained oligoanuria, severe metabolic acidosis, and drug toxicity. However, renal replacement therapy is often used for inborn errors of metabolism, hyperammonemia and uncontrolled hyperthermia, and tumor lysis syndrome. In pediatric patients receiving CRRT, like in adults, many factors are involved which can result in a greater mortality risk, e.g. vasopressor support, the need for mechanical ventilation, sepsis, the severity of the illness, contemporary failure of other organs, and a very high fluid overload [3, 4]. The most recent study [5] focusing on CRRT reported that survival in infants weighing less than $10 \mathrm{~kg}(43 \%)$ was lower than in children weighing more than $10 \mathrm{~kg}$ (64\%). No difference was found between children weighing less than $5 \mathrm{~kg}$ and those weighing 5-10 kg (44 vs. $42 \%$ ). Symons et al. [6] reported a poorer outcome of venovenous CRRT in patients under $3 \mathrm{~kg}$ compared to patients weighing between 3 and $10 \mathrm{~kg}$.

\section{Continuous Renal Replacement Therapy}

The provision of adequate CRRT to infants has been a historical challenge over the last 40 years. Since the continuous arteriovenous hemofiltration $(\mathrm{CAVH})$ era, initiated in the 70s by Kramer et al. [7], the need for an effective treatment has driven the search for adequate technologies. Thanks to a new minifilter and years of experience with adults, in 1981 the first infant was successfully treated [8]. New membranes and filters were specifically designed to obtain low-resistance extracorporeal circuits and ultrafiltration (UF) rates in the range of $1-3 \mathrm{ml} / \mathrm{min}$. Vascular access was obtained via cannulation of an artery and a vein with 6-french single-lumen catheters, achieving blood flows in the range of $12-25 \mathrm{ml} / \mathrm{min}$. The arteriovenous pressure gradient provided the force to move blood through the circuit, while no pumps were used either for blood circulation or for UF. Purification was mainly achieved via convection with a substitution solution as a replacement of ultrafiltrate. The main advantages of this technique were the low total circuit volume $(15 \mathrm{ml})$

A New Era of Renal Replacement

Therapies for Neonates and Small Infants achieved with short lines and a dedicated filter $(6 \mathrm{ml})$, the gentle fluid circulation ( $\mathrm{Qb} 20-80 \mathrm{ml} / \mathrm{min}$ ) which avoided hemodynamic perturbations, and the slow and continuous removal of fluids $(0.5-1.5 \mathrm{ml} / \mathrm{min})$.

Efficiency was progressively enhanced with refinement of the hemofilter geometry, the circuit and catheter designs, and the fluid balancing system, as well as the introduction of dialysis fluid.

A step forward was achieved with the introduction of pump-assisted continuous venovenous hemofiltration (CVVH), which was first described in adults in 1988 by Canaud et al. [9]. Advantages in terms of treatment performance, filter survival, and individual optimization of the 'dose therapy' were observed and CVVH rapidly became the gold-standard treatment. A few years later, in 1990, Yorgin et al. [10] initiated the 'adaptation era' for pediatric patients. Dialysis lines were cut using a sterile technique, reducing the extracorporeal circuit volume by 5-10 ml. Despite the undeniable advantages of these techniques, difficulties in the fine regulation of UF and unreliable continuous weight monitoring as a measure of UF were reported. Zobel et al. [11] noted, in an outcome study reporting both $\mathrm{CAVH}$ and $\mathrm{CVVH}$, that technical problems arose only with CVVH. More recently, in 19932001, Symons et al. [6] found very few complications using modern machinery. In a survey in 1999, CRRT was found to be the most common modality used for initial AKI treatment, increasing from 18 to $36 \%$, in contrast to PD which decreased from 45 to $31 \%$ in only 4 years [12].

\section{Circuits and Vascular Access}

Efficient vascular access has been, from the CAVH era, the crucial element determining the successful provision of CRRT. The shortness and large diameter of the cannulas were, in CAVH, essential to achieve a sustainable blood flow while avoiding unnecessary pressure losses. Several vascular accesses were promoted, including umbilical vessels and brachial artery or femoral artery cannulation.

In the adaptation era, in which adult CRRT machinery was used by fitting circuits to pediatric settings, a functional vascular access was mandatory to guarantee a blood flow capable of generating adequate access and return pressures to prevent related complications. A wide variety of acute vascular catheters has been described for the pediatric population, i.e. a single-lumen catheter using a 'single needle' has been placed in very low birth weight infants. Other strategies like the use of 2 single-lumen catheters in different central veins or use of the umbilical vein have also been 
Fig. 1. Diagram of the CARPEDIEM.

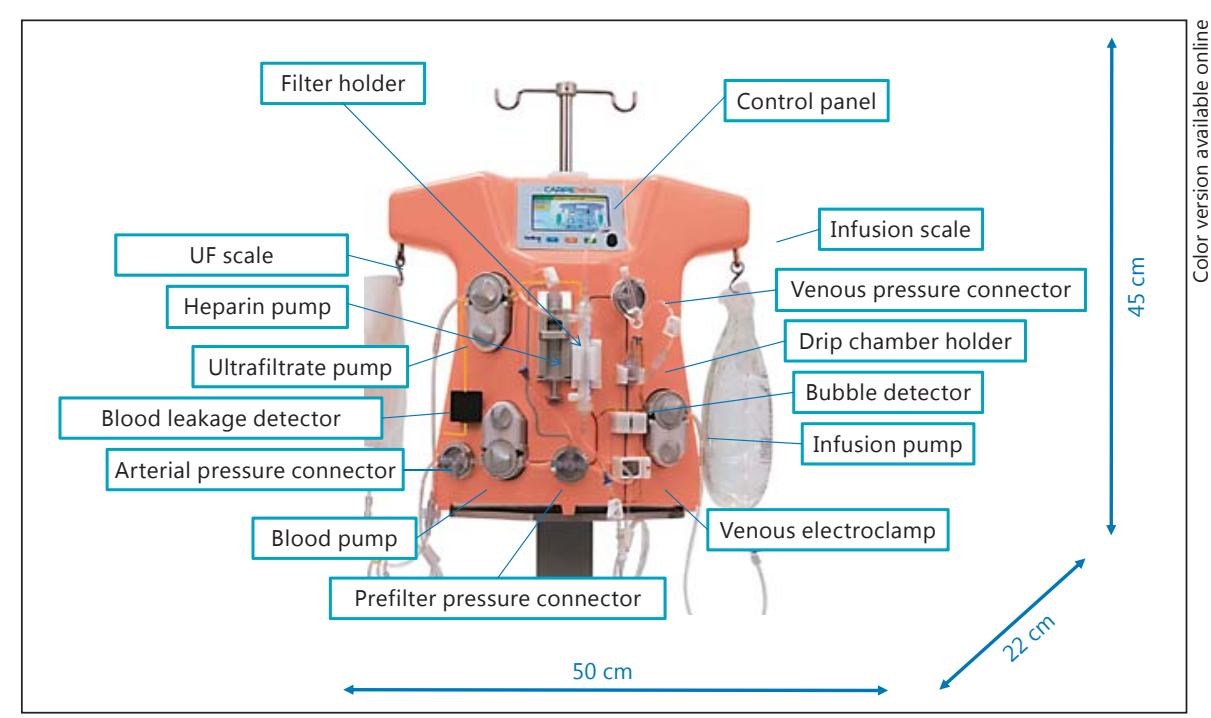

explored, in particular in patients weighing less than $5 \mathrm{~kg}$. A weight-related catheter size has been suggested [13].

A report of the Prospective Pediatric Continuous Renal Replacement Therapy Registry (ppCRRT) [14] examined the effect of vascular access size and location on circuit survival. The functional survival of the CRRT circuit increased significantly using larger catheters. The 48-hour survival with 8 -french catheters was $76 \%$, compared to $26 \%$ using 7 -french catheters. The survival time was less than $10 \mathrm{~h}$ with a 5 -french dual lumen. Jugular veins have a better survival than femoral or subclavian ones. Similar findings were reported by del Castillo et al. [15]. A prolonged filter life was associated with catheters larger than $6.5 \mathrm{~F}$, filters with a greater surface area $\left(>0.4 \mathrm{~m}^{2}\right)$, hemodiafiltration as treatment, and heparin levels above $10 \mathrm{IU} / \mathrm{kg} / \mathrm{h}$. An interesting finding was the slightly better filter life in children older than 12 months and with a weight greater than $10 \mathrm{~kg}$, although this difference was not statistically significant.

Unfortunately, the lengths of the catheters, as well as the blood flow and/or access and return pressures, were never taken into account. Catheterization with larger catheters may impair venous drainage and cause thrombosis; in addition, the circuit survival is shorter $[5,16,17]$.

\section{From ‘Adapted' to 'Fitted' Pediatric CRRT}

The most common strategy utilized nowadays to adapt adults machine to infants is to use blood or colloids to prime the extracorporeal circuit when its volume is greater than $10 \%$ of the child's circulating blood volume.
The following side effects due to the rapid infusion of citrated, hyperkalemic banked donor blood have been reported: acid-base and electrolyte imbalances and significant hemodynamic complications. In the past, bradykinin release syndrome also occurred due to exposure of the blood to the AN-69 membrane. An alternative technique requiring 2 machines with a so-called 'circuit-tocircuit exchange' in order to decrease blood exposure and minimize interruptions has also been proposed [18].

With the development of the modern CRRT machine, a dedicated circuit for pediatric use was proposed. A risk analysis showed that the dimension of the pumps and the accuracy of the UF system suggested a lower limit for the patient's weight. An accurate analysis of the machines widely used in the adult setting showed the balance error to be a worrisome 20-190 g [19]. Upper limits were set for the fluid balance error, related to the patient's weight, only with third generation machines. Typically the threshold for sudden errors in weight loss is 20 or $40 \mathrm{ml}$ (cumulative). In the presence of such technological limitations, adult machines were far from providing adequate and safe treatment to infants, newborns, and small children.

In 2012 the Cardio-Renal, Pediatric Dialysis Emergency Machine (CARPEDIEM) [20] (fig. 1) launched the 'fitted era' for pediatric CRRT. The project was born in the same place where 24 years earlier the first CAVH had been performed: the Department of Nephrology and International Renal Research Institute of the San Bortolo Hospital in Vicenza, Italy. The aim was to develop a CRRT platform specifically dedicated to newborns and small infants in the weight range of $2.0-10 \mathrm{~kg}$ and with an approximate BSA 
of $0.15-0.5 \mathrm{~m}^{2}$. Three circuits with surface areas of 0.075 , 0.15 , and $0.25 \mathrm{~m}^{2}$ are available. The 3 small roller pumps move blood into a $27-\mathrm{ml}$ ( 34 and $45 \mathrm{ml}$, respectively, with the bigger filters) total volume circuit. The solute clearance may reach 2 liters/h/1.73 $\mathrm{m}^{2}$ of BSA and/or 25$35 \mathrm{ml} / \mathrm{kg} / \mathrm{h}$ in patients weighing less than $10 \mathrm{~kg}$. To ensure a highly accurate fluid balance, the CARPEDIEM is equipped with gravimetric controls combined with an excellent scale sensitivity of $1 \mathrm{~g}$ for both infusion and effluent bags. Catheters (4-7 F) tested in vitro have shown excellent results in terms of circuit longevity [20]. The first in vivo application of the CARPEDIEM [21], done in Vicen$\mathrm{za}$ in a newborn weighing less than $3 \mathrm{~kg}$, seemed to confirm a paradigm shift in terms of the provision of adequate and highly safe CRRT to infants. A 4-french dual-lumen catheter has been used for more than $400 \mathrm{~h}$ in patients with multiple organ failure, fluid overload of $63 \%$, acidosis, hyponatremia, thrombocytopenia, and oliguria. No hypotension or complications related to CRRT were reported. The treatment led to the control of fluid balance, metabolic waste products, bilirubin, and other humoral disorders; the neonate recovered for multiple organ dysfunction was discharged from the hospital.

\section{Conclusions}

In the last decade, the indications for renal replacement in the pediatric population have changed to include a wide spectrum of diseases. The so-called 'nonrenal indications' have contributed to the increase in the number of CRRT applications mainly in critically ill infants and neonates. The renal replacement therapy of choice for this particular population is still PD. However, limitations in terms of efficiency call for the use of a more suitable therapy (i.e. one with higher UF rates and better solute clearance). CRRT may represent the adequate choice; nevertheless, the adequate technology to perform this therapy has never been developed. The CARPEDIEM, specifically designed for neonates and small infants, may represent the missing element in the pediatric renal replacement era.

\section{Disclosure Statement}

All authors declare no conflicts of interest.

\section{References}

1 Goldstein SL: Pediatric acute renal failure: demographics and treatment. Contrib Nephrol 2004;144:284-290.

2 Schneider J, et al: Serum creatinine as stratified in the RIFLE score for acute kidney injury is associated with mortality and length of stay for children in the pediatric intensive care unit. Crit Care Med 2010;38:933-939.

-3 Uchino S, et al: Acute renal failure in critically ill patients: a multinational, multicenter study. JAMA 2005;294:813-818.

4 Ostermann M, Chang RW: Correlation between parameters at initiation of renal replacement therapy and outcome in patients with acute kidney injury. Crit Care 2009; 13:R175.

5 Askenazi DJ, et al: Continuous renal replacement therapy for children $\leq 10 \mathrm{~kg}$ : a report from the prospective pediatric continuous renal replacement therapy registry. J Pediatr 2013;162:587-592.e3.

6 6 Symons JM, et al: Continuous renal replacement therapy in children up to $10 \mathrm{~kg}$. Am J Kidney Dis 2003;41:984-989.

7 Kramer P, et al: Arteriovenous haemofiltration: a new and simple method for treatment of over-hydrated patients resistant to diuretics. Klin Wochenschr 1977;55:1121-1122.
8 Ronco C, et al: Treatment of acute renal fail- 15 del Castillo J, et al: Circuit life span in critiure in newborns by continuous arterio-venous hemofiltration. Kidney Int 1986;29: 908-915.

-9 Canaud B, et al: Pump assisted continuous venovenous hemofiltration for treating acute uremia. Kidney Int 1988;24:S154-S156.

10 Yorgin PD, Krensky AM, Tune BM: Continuous venovenous hemofiltration. Pediatr Nephrol 1990;4:640-642.

$\checkmark 11$ Zobel G, et al: Five years experience with continuous extracorporeal renal support in paediatric intensive care. Intensive Care Med 1991;17:315-319.

12 Warady BA, Bunchman T: Dialysis therapy for children with acute renal failure: survey results. Pediatr Nephrol 2000;15:11-13.

13 Goldstein SL: Advances in pediatric renal replacement therapy for acute kidney injury. Semin Dial 2011;24:187-191.

14 Hackbarth R, et al: The effect of vascular access location and size on circuit survival in pediatric continuous renal replacement therapy: a report from the PPCRRT registry. Int J Artif Organs 2007;30:1116-1121. cally ill children on continuous renal replacement treatment: a prospective observational evaluation study. Crit Care 2008; 12:R93.

16 Goldstein SL, et al: Evaluation of the PRISMA M10 circuit in critically ill infants with acute kidney injury: a report from the Prospective Pediatric CRRT Registry Group. Int J Artif Organs 2006;29:1105-1108

17 Sohn YB, et al: Continuous renal replacement therapy in neonates weighing less than $3 \mathrm{~kg}$. Korean J Pediatr 2012;55:286-292.

18 Eding DM, et al: Innovative techniques to decrease blood exposure and minimize interruptions in pediatric continuous renal replacement therapy. Crit Care Nurse 2011;31:64-71.

19 Ronco C, et al: Management of fluid balance in CRRT: a technical approach. Int J Artif Organs 2005;28:765-776

20 Ronco C, Garzotto F, Ricci Z: CA.R.PE.DI.E.M. (Cardio-Renal Pediatric Dialysis Emergency Machine): evolution of continuous renal replacement therapies in infants - a personal journey. Pediatr Nephrol 2012;27:1203-1211.

21 Ronco C, et al: Renal replacement and extracorporeal blood therapies in neonates: a paradigm shift. Lancet 2014, in press.
A New Era of Renal Replacement

Therapies for Neonates and Small Infants 